\title{
Biological memory of childhood maltreatment - current knowledge and recommendations for future research
}

\author{
Prof. Dr. Iris-Tatjana Kolassa \\ Leiterin der Abteilung Klinische und Biologische Psychologie \\ Mitautoren: \\ Katharina Schury
}

\section{Kontakt:}

Prof. Dr. Iris-Tatjana Kolassa

Universität UIm

Fakultät für Ingenieurwissenschaften und Informatik

Institut für Psychologie und Pädagogik

Abt. Klinische und Biologische Psychologie

D-89069 Ulm

Telefon: $+49-(0) 731 / 5026590$

Telefax: +49-(0)731/50 26599

E-Mail: iris.kolassa@uni-ulm.de

URL: http://www.uni-ulm.de/index.php?id=29276 
Abstract: Child maltreatment (CM) leads to detrimental and lifelong psychological and physiological alterations - building a biological memory of $\mathrm{CM}$, which might even be transmitted to the next generation. After summarizing current knowledge of such biological consequences of $\mathrm{CM}$ (e.g. on the hypothalamus-pituitary-adrenal axis, on telomere length and telomerase activity, and the immune system), this article hints at important questions, that have to be answered in this research area. Therefore, we propose, that different research fields should join forces to gain a deeper understanding of the multiple effects of CM and especially on its transmission.

\section{Introduction}

Child maltreatment (CM) has detrimental effects on children's development with psycho-bio-social consequences throughout life. Furthermore, CM - and its consequences - can be transmitted to the next generation, leading to a "transgenerational cycle of maltreatment" (TCM). This article aims at describing existing knowledge on neurobiological determinants and sequelae of $\mathrm{CM}-\mathrm{a}$ biological memory of $\mathrm{CM}$. Furthermore, we will respond to possible transgenerational effects of CM. We do not attempt to systematically review all of the work in this widespread field. Rather, we want to connect knowledge from different fields of research - stress system, immune system, cell aging and epigenetics and stimulate research on this innovative and societal important topic.

$\mathrm{CM}$ includes sexual, physical and emotional abuse, as well as physical and emotional neglect (cf. ${ }^{1-3}$ ) and there is strong evidence that different forms of maltreatment are interrelated. ${ }^{4,5}$ Furthermore, the number of different types of maltreatment (e.g. beating, verbal humiliation) might be crucial for the development of consequential dysfunctions. We know from posttraumatic stress disorder (PTSD) - a psychiatric condition which can develop after traumatic experiences - that the risk for PTSD increases with the number of different traumatic event types experienced, i.e. with increasing traumatic load. ${ }^{6-8}$ Similarly, in CM a cumulative effect on developmental problems during adolescence and health in adult life can be supposed ${ }^{9,10}-$ an effect that could be described as maltreatment load.

Prevalence rates for maltreatment vary considerably due to methodological issues like differing definitions of maltreatment, variations in assessment methods (e.g. agency report, self-or parent-report) or samples used but also due to high estimated numbers of unreported cases. Cumulative prevalence rates between 5-35\% are reported for physical abuse, 4-9\% for severe emotional abuse, $15-30 \%$ for sexual abuse in girls and $5-15 \%$ in boys, and 6 $11.8 \%$ for neglect (cf. ${ }^{11}$.)

Common consequences of $\mathrm{CM}$ are mental health problems such as PTSD, depression, and anxiety, as well as cognitive distortions, risky sexual behavior, obesity, and criminal behavior ${ }^{11,12}$. Furthermore, maltreated children are reported to have poorer physical health throughout life including cardiovascular disease, diabetes, cancer, frequent headaches, accelerated cell aging and even 
premature mortality. ${ }^{13-15}$ Maltreatment might be especially detrimental in early childhood, as this time period is characterized by rapid neuroendocrinological development, which renders the brain and stress axes especially vulnerable to the influence of stress. ${ }^{16}$

Children of parents with a history of $\mathrm{CM}$ are at increased risk to experience $\mathrm{CM}$ themselves, ${ }^{17-20}$ leading to a transgenerational cycle of maltreatment, with transmission rates around $7 \% .^{17,21}$. However, importantly the majority of parents with own CM experiences do not maintain the cycle of maltreatment. ${ }^{17,22}$ Several risk and protective factors are known to play a role in TCM. Poor parenting styles, combined with parenting under 21 years, history of mental illness, and residing with a violent adult put families at increased risk for TCM, ${ }^{23}$ whereas financial stability, social support, a caring and stable caregiver appear protective. $^{17,24}$

To identify pre- and perinatal factors that play a role in the determinants and sequelae of $\mathrm{CM}$, as well as in the transmission of maltreatment to the next generation is an important step, which will finally help to advance interventions and services to families and children at risk.

\section{Biological mechanisms in CM-related disorders and in TCM}

To understand the development of a biological memory of $\mathrm{CM}$ as well as the transmission of $\mathrm{CM}$, mediating biological factors have to be taken into account. In the following, the state of research on alterations in neurobiological system (i.e. HPA axis, biomarkers of cell aging and the immune system) that are involved in the etiology of CM-related dysfunctions and in the transmission of maltreatment as well as epigenetic mechanisms in TCM will be characterized. A brief introduction to these biological systems will be given, followed by a narrative review of studies concerning the association of the particular system and $\mathrm{CM}$ as well as TCM, respectively.

\section{HPA axis}

The HPA axis is a key factor in the response to stress and leads to the release of the stress hormone cortisol. In response to stress, corticotropin releasing hormone $(\mathrm{CRH})$ is released from the hypothalamus, which stimulates the release of adrenocorticotropin releasing hormone $(\mathrm{ACTH})$ in the pituitary gland, resulting in the release of glucocorticoids (mostly cortisol) from the adrenal gland into blood circulation. The activity of the HPA axis is subject to a predictable circadian cycle and regulates itself by negative feedback loops - i.e. $\mathrm{CRH}$ and $\mathrm{ACTH}$ release is inhibited, when levels of blood cortisol are high. Temporarily elevated cortisol levels in response to an acute stressor are important for the organisms' adaption to environmental stressors and are needed to maintain homeostasis. However, childhood adversity seems to alter HPA axis activity and that these early changes persist. ${ }^{25}$ Chronically altered levels of cortisol increase the risk for later psychopathology, like depression 
(which is associated with hypercortisolism ${ }^{26}$ ) or PTSD (which is associated with hypocortisolism in patients with PTSD due to physical or sexual abuse ${ }^{27}$ ), but also for stress-related diseases like asthma or rheumatoid arthritis. ${ }^{28}$. In this context, the suppressing effect of cortisol on the immune system has to be mentioned, which can result in excessive inflammation.

Early life stress has been linked to abnormal functioning of the HPA axis in several investigations. But evidence of alterations in the HPA axis in the aftermath of $\mathrm{CM}$ is inconsistent (for a review $\mathrm{see}^{25}$ ). The conflicting results on HPA axis alterations after CM might stem from methodological issues like the operationalization of maltreatment, the sample (e.g. psychiatric patients vs. general population) or the collection and measurement of cortisol. ${ }^{29}$ So far, cortisol has mostly been measured in urine, blood or saliva. This measurements allow the assessment of cortisol over short time periods (minutes in blood or saliva, hours in saliva), but are subject to situational factors (e.g. food intake, perceived stress). In particular the circadian rhythm of cortisol production makes it difficult to compare results and the samples should be taken in accordance to a strict protocol. A new and promising approach to overcome these methodological problems is the analysis of cortisol in hair, in which cortisol levels can be estimated over long timer periods (approx. 3 months). ${ }^{30}$ The differing results on HPA axis activity subsequent to $\mathrm{CM}$ and/or trauma might also mirror the influence of other hormones, which are known to inhibit cortisol. The hormone dehydroepiandrosterone (DHEA) is a cortisol antagonist and protects the brain - in particular the hippocampus - from the damaging effect of cortisol and inhibits excessive inflammatory processes resulting from cortisol. ${ }^{31}$ When estimating HPA axis activity, one should not only rely on cortisol levels, but should consider using the ratio of cortisol and the cortisol antagonist DHEA. Studies have shown that the cortisol/DHEA ratio can serve as a more reliable marker of HPA axis activity (for an overview see ${ }^{31}$ ).

Despite conflicting results, one can reason, that $\mathrm{CM}$ leads to a dysregulation of the HPA axis, thereby impairing stress reactivity as well as health (through the influence of cortisol on inflammation) throughout the life of affected individuals. Even worse, alterations of the HPA axis might be transmitted to the next generation leading to a potential vulnerability of the infants stress system, but there are only few studies addressing transgenerational effects.. A 25-year longitudinal study observed lower morning cortisol as well as altered cortisol release during the course of the day compared to controls in children of mothers that reported social withdrawal in their childhood. ${ }^{32}$ As no cortisol levels of the mothers were assessed, developmental similarities in stress response patterns between mothers and their children can be supposed to exist but not confirmed in this study. In another study, lower baseline cortisol in infants of CM mothers were observed compared to control mother-infant pairs. ${ }^{33}$ Transgenerational effects of maternal stress caused by mental disorder on the HPA axis of their infants have also been confirmed in mothers with depression ${ }^{34,35}$ or PTSD. ${ }^{36,37}$ 
The HPA axis is the core system for stress regulation and impacts other important physiological systems, e.g. the immune system. An effect of maternal $\mathrm{CM}$ on the infants HPA axis would increase the risk for impaired stress adaption and stress-related illnesses in these children from the very beginning of their life. We need to understand the mechanisms of the transmission of altered HPA axis activity and develop interventions to circumvent that experiences of maltreatment not only impacts the life of a mother negatively, but also their children. More studies on the transmission of HPA axis dysregulations to the next generation are needed. These studies should try to overcome methodical problems, e.g. by strict measurement protocols, using the cortisol/DHEA ration to estimate HPA axis activity or by considering new methods, like the analysis of cortisol in hair.

\section{Cell aging}

Chronic and severe psychological stress can lead to premature cell aging as measured by telomere length and telomerase activity. ${ }^{38}$ Telomeric DNA is composed of repetitive DNA sequences (in humans TTAGGG), it caps the end of chromosomes and promotes chromosomal stability. In the process of somatic cell division, the telomere is not fully replicated and thus shortened at each replication ${ }^{39}$, finally leading to programmed cell death or senescence. ${ }^{40}$ The enzyme telomerase adds the necessary telomeric DNA and has direct telomere-protective functions. ${ }^{41}$ Telomere length and telomerase activity are accepted biomarkers for cell aging and longevity and shortened telomeres have been linked to several age related disorders like cardiovascular disease, cancer, diabetes ${ }^{42,43}$, and even all-cause mortality. ${ }^{44}$

In one of the first investigations on the effects of psychological stress on cell aging, women with high rates of chronic stress showed lower telomerase activity and accelerated telomere shortening compared to women with low stress levels. ${ }^{38}$ Shortened telomeres have in the meantime been confirmed in the aftermath of $\mathrm{CM}$ as well: One study observed shorter telomere length in mentally healthy adults with a history of maltreatment compared to nonmaltreated controls. ${ }^{45}$ In particular neglect or other adverse childhood experiences (ACE), like. parents' divorce, death of parent, which are usually seen as less detrimental than physical or sexual abuse can impact health through telomere shortening in adult life - with a difference of $640 \mathrm{bp}$ compared to individuals without adverse childhood experiences. ${ }^{46}$ Based on an average shortening rate of 31-63 bp per year, (average shortening rate across adulthood from 20-95 years; $\mathrm{cf}^{38}$ ) this can be translated into a 7- to 15-year difference in life span. Adults (with and without anxiety disorder), who reported ACE showed shorter telomeres adults without a history of ACE. ${ }^{47}$ Furthermore, a recent study on socially deprived children confirmed that accelerated telomere shortening can already be detected during childhood. ${ }^{48}$ O'Donovan et al. ${ }^{49}$ observed reduced telomere length subsequent to CM in PTSD patients. Importantly, the 
number of different categories of traumatic events in childhood was linearly associated with accelerated telomere shortening, indicating a cumulative effect of childhood trauma (maltreatment load). CM also accounted for the effect of PTSD on telomere length in this study. Only one study so far failed to confirm an association between $\mathrm{CM}$ and shorter telomere length ${ }^{50}$. This might be due to the rather superficial assessment of $\mathrm{CM}$, as participants were asked only five questions, if they had experienced physical, emotional or sexual abuse in childhood or later on, or due to the specific sample, as participants were only twins.

Research on accelerated telomere shortening in CM is still sparse and more research on the underpinnings of telomere shortening and possible transgenerational effects is needed. The role of telomerase activity has not yet been investigated in $\mathrm{CM}$.

\section{Immune system}

The immune system protects the body from pathogenic organisms like bacteria, viruses, fungus or defective cells of the own body. After tissue injury but also in response to stress, inflammatory processes are implemented by the immune system via the release of pro- and anti-inflammatory cytokines. But inflammatory processes have also been linked to the development of several chronic diseases such as cardiovascular, autoimmune, mental or neurodegenerative diseases. ${ }^{51-53}$

A large longitudinal study confirmed long-term effects of $\mathrm{CM}$ on adult inflammation: Individuals with a history of childhood maltreatment or social isolation had an increased risk for heightened inflammation (C-reactive protein level $>3 \mathrm{mg} / \mathrm{L}$ ) in adulthood, independent of the influence of additional early life stress, stress in adulthood or adult health and health behavior in this prospective study. ${ }^{54,55}$ In a study with 132 healthy older adults, including 58 dementia family caregivers, a history of multiple childhood adversities or childhood abuse was associated with elevated levels of proinflammatory cytokines. ${ }^{46}$ The reported relationship was more pronounced in caregivers compared to non-caregivers. Another study showed a greater overall peripheral release of the proinflammatory cytokine Interleukin (IL) 6, and elevated IL-6 concentrations during a stress test in individuals with $\mathrm{CM}$, compared to nonmaltreated controls. ${ }^{56}$

Potentially, traumatic experiences might also alter immune function via epigenetic changes: Uddin et al. (2010) demonstrated that a greater number of unmethylated genes led to changes in gene expression which were related to altered immune function.

We hypothesize that $\mathrm{CM}$ can also influence the immune system of the next generation- possibly via epigenetic pathways- leading to higher risks for illnesses in children of mothers with CM. One study showed, that children of mothers who were exposed to chronic interpersonal violence (during childhood 
as well as later on) had elevated IgE levels ${ }^{57}$ - which are associated with the development of allergies in later life. ${ }^{58}$ The mechanism underlying this transgenerational effect of $\mathrm{CM}$ on the immune system was not investigated in this study. We assume, that epigenetic pathways can explain such phenomena. Immunological "knowledge" seems to be epigenetically passed from the mother to her infant during limited neonatal imprinting periods (discussed further below) as well as during gestation with generally beneficial effects for the child's immune system. ${ }^{59-61}$ Despite the theoretical knowledge about such epigenetic transmission processes, there are hardly any studies addressing transgenerational pathways of immune alterations in $\mathrm{CM}$.

\section{The role of epigenetic alterations in maltreatment, trauma and the transmission of both}

Usually when speaking of "inheritance" one refers to the passing of genetic information from one generation to the next. ${ }^{62}$ However, not only the presence of genes but rather the level of gene expression determines individual variations in offspring phenotype. These levels of gene expression can be regulated by genetic polymorphisms, but there is mounting evidence that environmental effects can be transmitted to following generations via epigenetic alterations ${ }^{62}$, as was, for example, described for the immune system in the aforementioned study by Sternthal et al. (2009). ${ }^{57}$

Epigenetic mechanisms, i.e. alterations in chromatin structure such as DNA methylation, histone modifications, and RNA interference can occur in response to $\mathrm{CM}$ and might be involved in the transgenerational transmission of childhood maltreatment and traumatization. ${ }^{63,64}$ There are different pathways how epigenetic alterations in the offspring of CM mothers can be explained: First, stress during pregnancy might directly alter the epigenome of the child (e.g. ${ }^{65}$ ). Secondly, postnatal mother-infant interactions and low maternal care, which can be altered as a consequence of maltreatment experiences of the mother, can change the infant's epigenome. Alterations in the methylation status of specific gene sites were observed in rat pups as a result of low maternal care behavior as compared to high maternal care. This was shown for the glucocorticoid receptor gene promoter in the hippocampus ${ }^{66}$, the glutamic acid decarboxylase 1 (GAD1) promoter in the hippocampus ${ }^{67}$, and the brain-derived neurotrophic factor (BDNF) gene ${ }^{68}$. Cross-fostering seems to reverse the methylation changes associated with less attentive maternal care, emphasizing the ongoing importance of environmental influences and the possibility for interventions. ${ }^{66}$ There is also one study, which did not find alterations in the methylation status (in the glucocorticoid receptor promoter region) after early life adversity in rat pups, although abnormal behaviors, increased neurotrophin levels and HPA axis dysregulation were observed. ${ }^{69}$ And third, gametic transmission of epigenetic alterations can occur, as was investigated in male mice. Unpredictable and chronic separation from the mother altered DNA methylation 
in the promoter region of several candidate genes in the germline of these male mice. $^{70}$ The epigenome is largely erased during early preimplantation development of the germline and during germ cell development. However, some epigenetic marks are maintained, thus permitting gametic transmission of epigenetic alterations, making transgenerational epigenetic inheritance possible. ${ }^{71}$

Very few studies so far investigated transgenerational transmission of epigenetic changes subsequent to traumatic stress in humans. The first human study on this topic investigated influences of stress during pregnancy on the infant's epigenome. Exposure to depressed mood of the mothers in the third trimester of pregnancy was related to increased neonatal methylation levels at the glucocorticoid receptor promoter region. ${ }^{72}$ Interestingly, this increase of methylation levels was associated with an elevated stress responses of the HPA axis at three months after birth. Prenatal exposure to maternal depressed mood also affected the methylation patterns at the serotonin-transporter-linked polymorphic region (5-HTTLPR) ${ }^{73}$ And maternal gestational exposure to inter partnership violence influenced the methylation status in the promoter of the glucocorticoid receptor in the offspring even 10-19 years after birth. ${ }^{65}$ The few studies in humans point out that maternal stress during pregnancy can alter methylation patterns in their infants in affective- and stress-related gene sites (5-HTTLPR, glucocorticoid receptor gene), presumably leading to alterations in neurobiological systems in the infant. But evidence is still sparse and more and larger studies need to be implemented.

In investigating epigenetics, one has to keep in mind that results on epigenetic alterations measured in blood have to be interpreted with caution. Epigenetic changes are cell specific and it is so far unknown, how findings from the blood reflect changes in the brain. One study showed that childhood abuse leads to epigenetic changes in the brain in humans. ${ }^{74}$ In this study, alterations in the gene expression in the glucocorticoid receptor were found in the hippocampus of suicide victims with a history of child abuse compared to suicide victims without a history of child abuse and controls. Nevertheless, the issue of relating epigenetic findings in the blood with alterations in the brain remains open and has to be further addressed in humans and in animal models to gain a better understanding of the exact mechanisms of epigenetic transmission.

Epigenetics might be the key for understanding transgenerational transmission processes in $\mathrm{CM}$ and trauma. But knowledge from human studies is very limited and there are still methodological limitations that do not allow the generalization of study results (e.g. other influencing factors besides CM cannot be controlled in such studies). Nevertheless, the investigation of epigenetic alterations subsequent to $\mathrm{CM}$ and - most important - there transmission to the next generation seems to be a worthwhile undertaking. 


\section{Connecting and neurobiology and epigenetics}

The aforementioned neurobiological and epigenetic systems are closely linked to each other and to environmental factors (for illustration see figure 1).

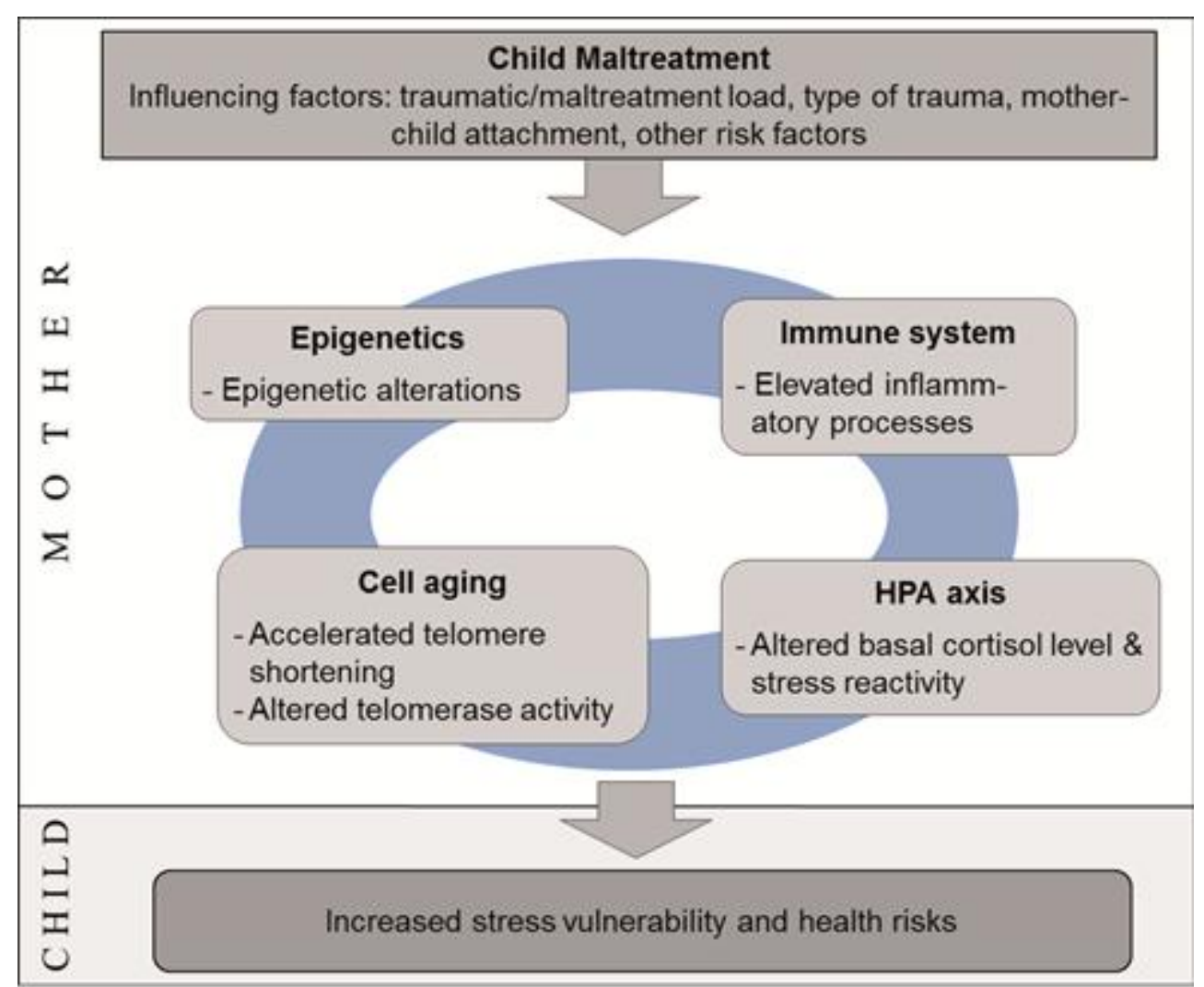

Figure 1 Schematic illustration of the association of core physiological systems that are altered subsequent to child maltreatment (cf. upper panel, mother), as well as the transmission of these alterations to the next generation (cf. lower panel, child). GR = Glucocorticoid receptor; HPA axis $=$ hypothalamus-pituitary-adrenal axis

Early life experiences can modulate the stress axis (i.e. HPA axis), thereby altering basal cortisol levels as well as the release of cortisol after a stressor. The stress response might also be influenced by epigenetic processes, e.g. by influencing the glucocorticoid receptor promoter $\left(\mathrm{cf}^{65}\right)$. Excessive activation of the body's stress systems can also accelerate telomere shortening, and thus, premature cell aging. Furthermore, the stress hormone cortisol is immunosuppressive. But under severe stress, cortisol can potentiate, instead of inhibiting, the activity of IL-1 and IL- $6{ }^{75}$, leading to elevated inflammation by impairing the functioning and regulation of the immune response to stress. Stress-related activation of the immune system might also be linked to telomere shortening and thereby an earlier onset of age-related diseases. Under acute and chronic stress, the number of immune cells increases and therefore the rate of cell division in immune cells rises. This increased rate of cell division results in a faster loss of telomere sequences. Moreover, cell culture studies have demonstrated that cortisol concentrations that are equal to physiological conditions suppress telomerase activity, which additionally leads to further 
telomere shortening. ${ }^{76}$ This highlights the importance of an interdisciplinary, multiperspective approach in studying mechanisms and consequences of $\mathrm{CM}$ and TCM, respectively. Joining forces in the field of $\mathrm{CM}$ and investigating the psychological mechanisms, risk and protective factors in $\mathrm{CM}$ and relating them to the various biological systems is necessary in future studies. Especially longitudinal designs which investigate psychological and biological functioning at the same time are necessary to gain a deeper understanding of the mechanisms in CM and TCM and finally to develop tailored early interventions for families and children at risk.

\section{Literature}

1. Briere, J. 1992. Child abuse trauma: Theory and treatment of the lasting effects. CA: SAGE, Newbury Park.

2. Cicchetti, D. \& Toth, S.L. 2005. Child maltreatment. Annu rev clin psychol. 1: 40938.

3. Myers, J.E.B. et al. 2002. APSAC Handbook on Child Maltreatment. CA: SAGE, Thousand Oaks.

4. Dong, M. et al. 2004. The interrelatedness of multiple forms of childhood abuse, neglect, and household dysfunction. Child abuse negl . 28: 771-84.

5. Green, J.G. et al. 2010. Childhood adversities and adult psychiatric disorders in the national comorbidity survey replication I: Associations with first onset of DSM-IV disorders. Arch gen psychiatry. 67: 113-23.

6. Kolassa, I.-T. et al. 2010a. The risk of posttraumatic stress disorder after trauma depends on traumatic load and the catechol-o-methyltransferase Val(158)Met polymorphism. Biol psychiatry. 67. 304-8.

7. Kolassa, I.-T. et al. 2010b. Spontaneous remission from PTSD depends on the number of traumatic event types experienced. Psychological Trauma: Theory, Research, Practice, and Policy. 2: 169-74.

8. Neuner, F. et al. 2004. Psychological trauma and evidence for enhanced vulnerability for posttraumatic stress disorder through previous trauma among West Nile refugees. BMC Psychiatry. 4: 34.

9. Anda, R.F. et al. 2006. The enduring effects of abuse and related adverse experiences in childhood. A convergence of evidence from neurobiology and epidemiology. Eur arch psy clin n. 256: 174-86.

10. Duke, N.N. et al. 2010. Adolescent violence perpetration: Associations with multiple types of adverse childhood experiences. Pediatrics. 125: e778-86.

11. Gilbert, R. et al. 2009. Burden and consequences of child maltreatment in highincome countries. Lancet. 373: 68-81. 
12. Briere, J. \& Jordan, C.E. 2009. Childhood maltreatment, intervening variables, and adult psychological difficulties in women: An overview. Trauma violence abuse 10: 375-88.

13. Anda, R.F. et al. 2010. Adverse childhood experiences and frequent headaches in adults. Headache. 50: 1473-81.

14. Brown, D.W. et al. 2009. Adverse childhood experiences and the risk of premature mortality. Am j prev med. 37: 389-96.

15. Felitti, V.J. et al. 1998. Relationship of childhood abuse and household dysfunction to many of the leading causes of death in adults. The Adverse Childhood Experiences (ACE) Study. Am j prev med. 14: 245-58.

16. Panzer, A. 2008. The neuroendocrinological sequelae of stress during brain development: The impact of child abuse and neglect. Afr J Psychiatry. 11: 29-34.

17. Dixon, L. et al. 2009. Patterns of Risk and Protective Factors in the Intergenerational Cycle of Maltreatment. J Fam Violence. 24: 111-22.

18. Pears, K.C. \& Capaldi, D.M. 2001. Intergenerational transmission of abuse: A twogenerational prospective study of an at-risk sample. Child Abuse Neglect. 25: 143961.

19. Sidebotham, P. \& Golding, J. 2001. Child maltreatment in the "children of the nineties" a longitudinal study of parental risk factors. Child Abuse Neglect. 25: 1177200.

20. Berlin, L.J. et al. 2011. Intergenerational continuity in child maltreatment: Mediating mechanisms and implications for prevention. Child Dev. 82: 162-76.

21. Browne, K.D. \& Herbert, M. 1997. Preventing Family Violence. Wiley: Chichester.

22. Kaufmann, J. \& Ziegler, E. 1989. The intergenerational transmission of child abuse. Child maltreatment: Theory and research on the causes and consequences of child abuse and neglect, pp. 129-150. Cambridge University Press. Cambridge.

23. Dixon, L. et al. 2005. Risk factors of parents abused as children: a mediational analysis of the intergenerational continuity of child maltreatment (Part I). $J$ Child Psychol Psychiatry. 46: 47-57.

24. Kaufmann, J. \& Henrich, C. 2000. Exposure to violence and early childhood trauma. In: Handbook of infant mental health, pp. 195-207. Guilford Press. New York.

25. Hunter, A.L. et al. 2011. Altered stress responses in children exposed to early adversity: A systematic review of salivary cortisol studies. Stress. Early Online: 1-3.

26. Holsboer, F. 2001. Stress, hypercortisolism and corticosteroid receptors in depression: Implications for therapy. J Affect Dosorders. 62: 77-91.

27. Meewisse, M.-L. et al. 2007. Cortisol and post-traumatic stress disorder in adults: systematic review and meta-analysis. British J Psychiatry. 191: 387-92. 
28. Heim, C. et al. 2000. The potential role of hypocortisolism in the pathophysiology of stress-related bodily disorders. Psychoneuroendocrino. 25: 1-35.

29. Cicchetti, D. \& Rogosch, F.A. 2001. The impact of child maltreatment and psychopathology on neuroendocrine functioning. Dev Psychopathol. 13: 783-804.

30. Gow, R. et al. 2010. An assessment of cortisol analysis in hair and its clinical applications. Forensic Sci Int. 196: 32-7.

31. Guilliams, T.G. \& Edwards, L. 2010. Chronic stress and the HPA axis: Clinical assessment and therapeutic considerations. Standard 9: 1-12.

32. Fisher, D.B. et al. 2007. Intergenerational predictors of diurnal cortisol secretion in early childhood. Infant Child Dev. 170: 151-70.

33. Brand, S.R. et al. 2010. The impact of maternal childhood abuse on maternal and infant HPA axis function in the postpartum period. Psychoneuroendocrino. 35: 68693.

34. Brennan, P.A. et al. 2008. Maternal depression and infant cortisol: influences of timing, comorbidity and treatment. J Child Psychol Psyc. 49: 1099-1107.

35. Laurent, H.K. et al. 2011. Risky shifts: How the timing and course of mothers' depressive symptoms across the perinatal period shape their own and infant's stress response profiles. Dev Psychopathol. 23: 521-538.

36. Yehuda, R. et al. 2007. Parental posttraumatic stress disorder as a vulnerability factor for low cortisol trait in offspring of holocaust survivors. Arch Gen Psychiatry. 64: 1040-8.

37. Yehuda, R. et al. 2005. Transgenerational effects of posttraumatic stress disorder in babies of mothers exposed to the World Trade Center attacks during pregnancy. $J$ Clin Endocrinol Metab. 90: 4115-8.

38. Epel, E.S. et al. 2004. Accelerated telomere shortening in response to life stress. $P$ Natl Sci USA. 101: 17312-5.

39. Chan, S.R.W.L. \& Blackburn, E.H. 2004. Telomeres and telomerase. Philos Trans $R$ Soc Lon B Biol Sci 359: 109-21.

40. Jiang, H. et al. 2008. Proteins induced by telomere dysfunction and DNA damage represent biomarkers of human aging and disease. P Natl Acad Sci USA. 105: 11299-304.

41. Chan, S.R.W.L. \& Blackburn, E.H. 2003. Telomerase and ATM/Tel1p protect telomeres from nonhomologous end joining. Molecular cell. 11: 1379-87.

42. Fitzpatrick, A.L. et al. 2007. Leukocyte telomere length and cardiovascular disease in the cardiovascular health study. Am J Epidemiol. 165: 14-21.

43. Willeit, P. et al. 2010. Telomere length and risk of incident cancer and cancer mortality. JAMA 304: 69-75. 
44. Cawthon, R.M. et al. 2003. Association between telomere length in blood and mortality in people aged 60 years or older. Lancet. 361: 393-5.

45. Tyrka, A.R. et al. 2010. Childhood maltreatment and telomere shortening: Preliminary support for an effect of early stress on cellular aging. Biol Psychiatry. 67: 531-34.

46. Kiecolt-Glaser, J.K. et al. 2010. Childhood adversity heightens the impact of laterlife caregiving stress on telomere length and inflammation. Psychosom Med. 73: 1622.

47. Kananen, L. et al. 2010. Childhood adversities are associated with shorter telomere length at adult age both in individuals with an anxiety disorder and controls. PLoS One. 5: e10826.

48. Drury, S.S. et al. 2011. Telomere length and early severe social deprivation: Linking early adversity and cellular aging. Mol Psychiatry. Epub ahead of print: 1-9.

49. O'Donovan, A. et al. 2011. Childhood trauma associated with short leukocyte telomere length in posttraumatic stress disorder. Biol Psychiatry. 70: 465-71.

50. Glass, D. et al. 2010. No correlation between childhood maltreatment and telomere length. Biol Psychiatry. 68: e21-e22.

51. Dantzer, R. et al. 2008. Frim inflammation to sickness and depression: When the immune system subjugates the brain. Nat Rev Neurosci. 9: 46-56.

52. McGeer, P.L. \& McGeer, E.G. 2004. Inflammation and the degenerative diseases of aging. Ann NY Acad Sci. 1035: 104-16.

53. Yudkin, J.S. et al. 2000. Inflammation, obesity, stress and coronary heart disease: Is interleukin-6 the link? Atherosclerosis. 148. 209-14.

54. Danese, A. et al. 2009. Adverse childhood experiences and adult risk factors for age-related disease: Depression, inflammation, and clustering of metabolic risk markers. Arch Pediatr Med. 163: 1135-43.

55. Danese, A. 2007. Childhood maltreatment predicts adult inflammation in a lifecourse study. Pro Natl Acad Sci USA. 104: 1319-24.

56. Carpenter, L.L. et al. 2010. Association between plasma IL-6 response to acute stress and early-life adversity in healthy adults. Neuropsychopharmacology. 35: 2617-23.

57. Sternthal, M.J. et al. 2009. Maternal interpersonal trauma and cord blood IgE levels in an inner-city cohort: A life-course perspective. J Allergy Clin Immunol. 124: 95460 .

58. Halken, S. 2003. Early sensitisation and development of allergic airway disease risk factors and predictors. Paediatr Respir Rev. 4: 128-34.

59. Lemke, $\mathrm{H}$. et al. 2009. Benefits and burden of the maternally-mediated immunological imprinting. Autoimmun Rev. 8: 394-9. 
60. Wright, R.J. 2007. Prenatal maternal stress and early caregiving experiences: Implications for childhood asthma risk. Paediatr Perinatal Epidemiol. 21 (Suppl. 3): 8-14.

61. Peden, D.B. 2000. Development of atopy and asthma: Candidate environmental influences and important periods of exposure. Environ Health Perspect. 108 (Suppl. 3): 475-82.

62. Champagne, F. A. 2008. Epigenetic mechanisms and the transgenerational effects of maternal care. Front Neuroendocrinol. 29: 386-97.

63. Arai, J.A. \& Feig, L.A. 2010. Long-lasting and transgenerational effects of an environmental enrichment on memory formation. Brain Res Bull. 85 (1-3): 30-5.

64. Turner, B. 2001. Chromatin and gene regulation. Blackwell Science Ltd. Oxford.

65. Radtke, K.M. et al. 2011. Transgenerational impact of intimate partner violence on methylation in the promoter of the glucocorticoid receptor. Transl Psychiatry. 1: e.

66. Weaver, I.C.G. et al. 2004. Epigenetic programming by maternal behavior. Nat Neurosci. 7: 847-54.

67. Zhang, T.-Y et al. 2010. Maternal care and DNA methylation of a glutamic acid decarboxylase 1 promoter in rat hippocampus. J Neurosci. 30: 13130-7.

68. Roth, T.L. et al. 2009. Lasting epigenetic influence of early-life adversity on the BDNF gene. Biol Psychiatry. 65: 760-9.

69. Daniels, W. et al. 2009. Maternal separation alters nerve growth factor and corticosterone levels but not the DNA methylation status of the exon 17 glucocorticoid receptor promoter region. Metab Brain Dis. 24: 615-27.

70. Franklin, T.B. \& Mansuy, I.M. 2010. Epigenetic inheritance in mammals: evidence for the impact of adverse environmental effects. Neurobiol Dis. 39: 61-5.

71. Lange, U.C. \& Schneider, R. 2010. What an epigenome remembers. BioEssays. 32 : 659-68.

72. Oberlander, T.F. et al. 2008. Prenatal exposure to maternal depression, neonatal methylation of human glucocorticoid receptor gene (NR3C1) and infant cortisol stress responses. Epigenetics. 3:97-106.

73. Devlin, A.M. et al. 2010. Prenatal exposure to maternal depressed mood and the MTHFR C677T variant affect SLC6A4 methylation in infants at birth. PloS one. 5: e12201.

74. McGowan, P.O. et al. 2009. Epigenetic regulation of the glucocorticoid receptor in human brain associates with childhood abuse. Nat Neurosci. 12: 342-8.

75. Dhabhar, F.S. \& McEwen, B.S.2001. Bidirectional effects of stress and glucocorticoid hormones on immune function: Possible explanations for paradoxical observations. In R. Ader, D.L. Felton \& N. Cohen (Eds.) Psychoneuroimmunology Third Edition. Academic Press, 301-38. 
76. Choi, J. et al. 2008. Reduced telomerase activity in human T lymphocytes exposed to cortisol. Brain Behav Immun. 22: 600-5. 